\title{
Medical apps in need of optical microspectrometers
}

\author{
Reinoud F. Wolffenbuttel ${ }^{1}$ Tanya M. Wolffenbuttel Hosli ${ }^{2}$
}

Received: 31 August 2015 / Accepted: 7 December 2015 / Published online: 30 January 2016

(c) The Author(s) 2016. This article is published with open access at Springerlink.com

\begin{abstract}
Apps have been used for providing additional user functionality on demand to the smartphone. Several highly promising medical apps have been introduced that rely on the build-in camera. However, some of these apps fall short of the expectations, due to the inherent limitation of the camera to operation in the visible spectral range. The more detailed spectral information that an optical microspectrometer can offer is needed. Although CMOS-compatible MEMS technologies enable the on-chip integration of the microspectrometer with the CMOS camera, implementing such a step would result in a device that would be considered a customized unit within a universal platform. Consequently, the economic viability would depend on the added value of the microspectrometer in popular applications versus the intrusion of the microspectrometer-specific processing on the imager fabrication. The LVOF-based microspectrometer is introduced as a CMOScompatible concept to meet the challenges imposed by the high production volume that is characteristic for the smartphone. The state-of-the-art of both the interactive optical app and the LVOF-based microspectrometer are presented.
\end{abstract}

\section{Introduction}

A wide range of apps is presently available for the user of a smartphone. The most simple and most widespread type of

Reinoud F. Wolffenbuttel

R.F.Wolffenbuttel@tudelft.nl

1 Electronic Instrumentation Laboratory, Microelectronics Department, Faculty of EEMCS, Delft University of Technology, Mekelweg 4, 2628 CD Delft, The Netherlands

2 Maartenscollege, Aart van der Leeuwkade 14, 2274 KX Voorburg, The Netherlands app provides information. This could be as basic as a map or catalog for general use, but could also be as specific as a listing of medications to be used by the medical practitioner. A more versatile type of app is the interactive app where typically some of the information that is provided by sensors within the smartphone is used. In many of such apps the camera is the core part, such as the fingerprint reader, but more complex apps use other sensors or combination of data sources, such as the build-in accelerometer in combination with GPS in apps for determining the level of physical activity of the user. The interactive aspect of the interactive app is in the feedback that it provides to the personal health-concerned user. In this physical activity monitoring app there is no reason for concern about misuse, as the user is unlikely to develop an unhealthy (of even dangerous) level of physical activity due to information provided by the app. However, in general there is a risk in the use of interactive medical apps that have been developed for medical purposes of for assisting a healthy lifestyle.

An interactive medical app provides in principle the opportunity to make a medical diagnosis or to provide incentives to a user to adapt to a lifestyle that is presumably more healthy, without consultation of the official medical practitioner, thus effectively by-passing the medical infrastructure. Any conclusion taken from data provided by e.g. the camera on melanoma using a skin cancer detection app (https://www.skinvision.com/en) or glaucoma using an eye retina app (http://www.peekvision.org/what-it-does (retina app)) has a limited confidence of being correct. In principle this is not unlike the diagnosis by the medical practitioner, who is also prone to making human error. There are two error modes: the first is the incorrect detection of the presumed disease (alpha risk, also referred to as false positive or type 1 error), while the second is missing the disease where the asp is intended for, when the disease is actually 
present (beta risk, also referred to as false negative or Type 2 error). The probability of occurrence of either error mode should of course be minimized. The first type of error may cause significant temporary emotional distress to the user. However, the rational response is to contact the medical practitioner as soon as possible and the long-term effects should be limited. The second type of error is more worrisome. False re-assurance may keep the patient away from the hospital until it may be too late for effective treatment.

Of course the problems associated with self-diagnosis were already applicable to the medical encyclopedia that have been available to the general public for generations. However the interactive medical app has significantly increased the problem. Firstly, because of the extensive use of the smartphone by the general public. Secondly, policymakers have started relatively recently to promote the use of technology that has become available for personalized systems (like the smart phone) for developing personalized medical monitors in an effort to reduce the cost of healthcare in an ageing population. This ambition has been translated into significant research funding by both the NIH in the USA and the EU (http://ec.europa.eu/ research/participants/data/ref/h2020/wp/2014_2015/main/ h2020-wp1415-health_en.pdf).

Such an ambition also brings a responsibility and it is not surprising that a discussion has started between the medical society and policymakers about what should be the definition of a 'medical device' and what should be the requirements to be imposed on a smartphone and an app to make it qualify as a 'medical device'. These concerns are being studied (http://www.ncbi. nlm.nih.gov/pmc/articles/PMC3959919/). The FDA has made steps towards regulation (http://www.regulations. gov/\#!documentDetail;D=FDA-2011-D-0530-0001 (also http://www.fda.gov/downloads/ForConsumers/ConsumerUpdates/UCM263405.pdf)). A key aspect is the embedding of the system within the medical infrastructure (i.e. the app should be for assistance and pre-screening and, therefore, in professional use linked to the medical practitioner and the patient database-which of course generates privacy issues), but should also satisfy 'reasonable' standards for acceptable alpha and beta risk.

Improving the quality of the sensory information on which an app is based, therefore, improves acceptance of use of apps, especially in medical applications. The stateof-the-art spatial resolution of the image is typically not a limiting factor, as is demonstrated by the success of the retina app, especially in remote parts of the world (http:// www.sciencedaily.com/releases/2014/10/141020084751. $\mathrm{htm})$. However, in several of the applications where the app could be of high impact [such as the app-based melanoma detection (https://www.skinvision.com/en)], some of the essential spectral information is not contained within the visible/near-infrared spectral range that is covered by the state-of-the-art pixel in the CMOS imager. Hyperspectral imager have the potential to overcome this limitation (Martin et al. 2014). However, these highly specific devices are unlikely to be included as a general-purpose component in any future smartphone, which is almost by definition intended for a high-volume market. As is demonstrated in this paper, detailed spectral analyses beyond the visible spectral range can be achieved in some cases by including a microspectrometer to (part of) the CMOS camera that is already part of the smartphone. Such an objective relies heavily on the technology for CMOS compatible microspectrometer fabrication. The business case of this concept is that available semi-medical apps, such as the measuring UV index of sun exposure (http://www.esrl.noaa.gov/ gmd/grad/neubrew/docs/UVindex.pdf) and the melanoma app (https://www.skinvision.com/en), may have enticed the public and adding more hardware in their smartphone (which is at the moment already packed with sensors) for a more reliable outcome may be an appealing feature, provided that this adds only marginally to the overall system costs. Non-medical uses of a microspectrometer in a smartphone may include the measurement of plant photosynthesis (PAR) in agriculture.

MEMS-based microspectrometers are typically composed of MEMS devices for operation as dispersive element or as tunable resonator within an optical system that also includes collimating optics, detector and, in some applications, an excitation light source. Suitable microspectrometers should fully exploit the benefits of functional integration of MEMS structure and the optics, while also enable the merging with detector (arrays) and circuits in CMOS.

The actual exploitation of the functional integration of the entire microspectrometer with all its core components within one functional unit (the IC-compatible package oreven more challenging - the chip) imposes several research challenges. The first is the general challenge of the fabrication compatibility of the MEMS processing and detector fabrication with CMOS technology. The second challenge is specific to the microspectrometer and is about the need for a minimum optical path length in diffractive optics to meet demanding optical specifications.

\section{CMOS-compatible MEMS-based microspectrometers}

Initial research was mainly on the use of MEMS technology for the fabrication of gratings (Keller et al. 2000). Successful devices have been realized for as wide spectral range [down to EUV wavelengths, with suitable deposited metals (Lin et al. 2011)]. 
It should be noted that miniaturization is difficult to combine with high-performance diffractive optics (Wolffenbuttel 2005). Projection of a dispersed spectrum onto a detector array simply requires an optical path of sufficient length. Obviously, mirrors can be co-integrated, but that would be at the expense of system complexity. This issue has resulted in a preference for filter/resonatorbased devices in microspectrometer research, despite the potential for higher optical throughput of the grating-based spectrometer, because the wavelength selective part can be positions directly on top of the detector. The first successful microspectrometer was the MEMS-based Fabry-Perot (FP) etalons for use in channel switching in fiber communication (Jerman et al. 1991). The convenient fact that silicon is transparent in the intended spectral range $(1550 \mathrm{~nm})$ was exploited. However, electrostatically tunable FP devices have also been designed for use in the visible spectral range.

Another approach is to implement the dispersion of incident light within a waveguide on top of a silicon wafer. Fibre technology and silicon micromachining are combined to yield a planar waveguide based grating spectrometer. The light to be analysed is introduced into the polymer waveguide through an optical fibre, is dispersed in a reflection grating that has been fabricated using deep reactive ion etching of the polymer, and projected onto an array of fibres that guide the spectral components to an array of photodetectors (Mohr et al. 1991).

A highly promising direction in waveguide-based microspectrometer design is the use of photonic crystal structures (Tanabe et al. 2007). Laterally changing the pitch or size of a dense regular array of holes or tubes that pattern the waveguide by design does provide a laterally defined filtering of light propagating through the waveguide. Extremely high quality factors are in principle possible $\left(\mathrm{Q}>10^{6}\right)$. However, this approach is restricted to a relatively narrow free spectral range. Moreover, tunability remains a challenge. Nevertheless, waveguide-based systems are uniquely suited for optical channel switching in a narrowband. In this paper the emphasis is on microspectrometer that are not subject to the fundamental limitations of the waveguide.

Silicon was in the initial phase of optical MEMS technology development mainly used as a mechanical material and the availability of micromachining techniques for the precise definition of the features (Correia et al. 1999). Using micromachining technologies for fabrication of entire optical systems has proven difficult to achieve, which was mainly because of the incompatibility of planar technology with high-quality spectrometers. The best that can be achieved with a planar grating-based microspectrometer is a resolution of about $10 \mathrm{~nm}$ over the $630-730 \mathrm{~nm}$ spectral range (Grabarnik et al. 2007), which can be significantly

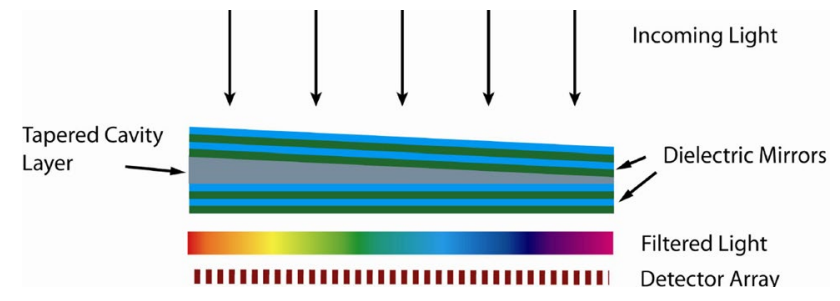

Fig. 1 A tapered Fabry-Perot LVO filter for filtering the collimated light (Emadi et al. 2012)

\section{Dielectric Mirror \\ Detector array}

Cavity Layer
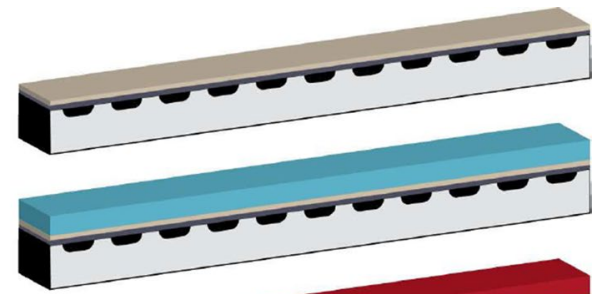

Photoresist

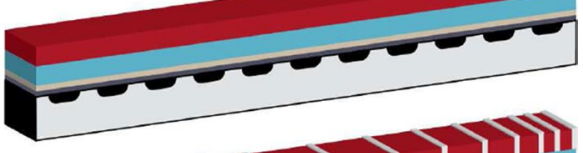

Photoresist

Patterned

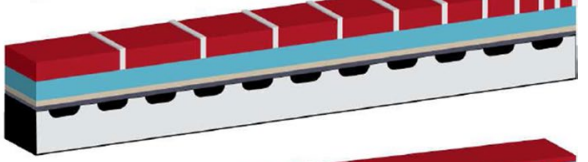

Photoresist

Reflow

Dry etching

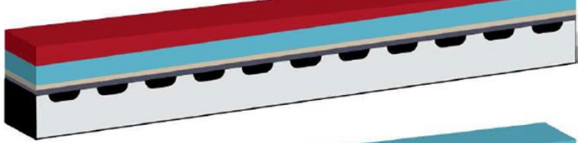

Dielectric Mirrors

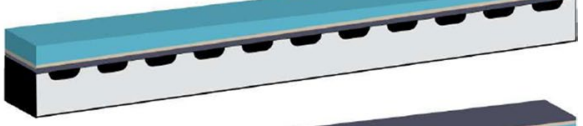

Dielectric Mirrors

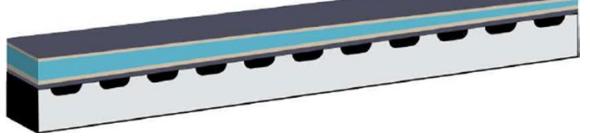

Fig. 2 Process flow for fabrication of linear variable optical filters (Emadi et al. 2009)

improved at the expense of on-chip CMOS-compatibility using an optical design with an external spherical mirror (Grabarnik et al. 2008).

A highly successful concept for narrowband spectroscopy is the Linearly variable Optical Filter (LVOF) (Emadi et al. 2012). As the tapered FP filter is fabricated directly on top of a detector array using low-temperature processing on cleanroom-compatible materials, CMOS-compatibility is possible for on-chip functional integration. The incident light spot should be imaged over the entire detector array, resulting is a poor optical throughput in general purpose, wideband applications, as compared to gratingbased microspectrometer systems. However, many promising application involve spectral analysis in a narrow band 
around an absorption line or luminescence line, which would favor the LVOF concept with typically 40-80 channels within this band. Particularly promising are applications in which several absorption lines need to be analyzed simultaneously, since multiple LVOFs can be fabricated simultaneously on a chip using a specially designed mask and reflow to result in tapered layers with different taper angles.

The LVOF is basically a one-dimensional array of many Fabry-Perot (FP)-type of optical resonators. Rather than a huge number of discrete devices, the LVOF has a center layer (the resonator cavity) in the shape of a strip and a thickness that changes over its length, as shown in Fig. 1. Dielectric mirrors are on either side. Thus the narrow passband wavelength of the LVOF varies linearly along its length. A detector array positioned underneath the LVOF records the spectrum of the projected light.

CMOS-compatible LVOF fabrication is based on the reflow of a specially patterned layer of resist (Emadi et al. 2009). Figure 2 shows the basic process sequence for fabrication. The process starts by deposition of the lower dielectric mirror stack and the oxide layer that results in the cavity layer. Photoresist is spin coated as the next step and lithography is applied to define the strip-like structure in the resist layer to be reflowed. A series of trenches of constant width and with variable pitch or trenches of variable width and constant pitch are etched over the length of the strip of resist to vary the effective amount of resist per unit area. The subsequent reflow transfers this gradient volume of resist into a smooth tapered resist layer. The tapered shape of the resist layer is transformed into the thick oxide cavity layer by an appropriate plasma etching process. The process is completed with the deposition of the top dielectric mirror stack.

The state-of-the-art demonstrates that fully integrated devices are possible. The most promising applications of fully integrated LVOF-based microspectrometers are those in which low unit cost are decisive and for which very specific requirements apply to the spectrum to be analyzed. In general the expected applications would be in the consumer market.

\section{Potential of microspectrometers in apps}

The expected future trends in application of CMOS compatible MEMS technology is, obviously, largely defined by the fields in which it can bring significant benefits. In general there are two different persuasive arguments for the introduction of CMOS-compatible MEMS technology: (1) the cost benefit per unit (chip) that the batch processing of silicon wafers can bring and (2) the merits extreme miniaturization. These also apply to optical MEMS technology.
The low unit cost in batch fabrication is widely recognized as the main driver of mainstream IC technology and also applies to MEMS (Wolffenbuttel 1996). The smartphone has proven to be a personal device for experimenting with sensors of which not all are, at least at the moment of their introduction at the market, obviously useful to the user. The particular strength of the smartphone concept is the fact that it can be considered a generic platform that contains hardware components to be programmed on user demand to perform a requested function. Therefore, in many circumstances the introduction of apps makes the already available sensors useful. The smart phone market is a high-volume market and is possibly succeeded/supplemented by the smart watch. There are several applications of significant public interest that involve spectral analysis. The performance obtained with sensors that are available (primarily the imager) has not always been satisfactorily for that purpose, which suggests a high-volume market for a simple, low-cost CMOS-compatible microspectrometer in every smartphone.

The probability that any type of microspectrometer would ever become a part of a future smart phone depends on the number and perceived importance of the applications it could serve and the extent in which the already implemented in-system sensors cannot provide a satisfactorily solution. A first example is an app for measuring highly localized skin discoloring and providing a warning in case the image of a particular part of the skin would suggest a risk. A typical 'melanoma alert' app is SkinVision (https://www.skinvision.com/en). The operation is based on an analysis of the microstructure of the skin using a photo that taken with the imager on the smartphone. There are two modes of operation: an instant mode of operation, in which the photograph of the suspected spot is analyzed using custom algorithm and a general database, and a repetitive mode in which a series of photographs is taken over a longer duration, which are recorded and analyzed for changes. Obviously, the second mode of operation is more useful, but less compatible with an understandable desire for 'immediate reassurance'. However, too high a rate of incorrect results are reported (a mix of $\alpha$ and $\beta$ type), which is not acceptable (http://www.npr.org/sections/ health-shots/2013/01/16/169524178/skin-doctors-question-accuracy-of-apps-for-cancer-risk). Of course the performance can be steadily improved by software development. However, a more fundamental solution would be to include additional information. One approach for improvement is to use a fluorescence of the tissue as the input signal (Das et al. 2015). However, this technique requires an optical excitation source, which is difficult to include in a general-purpose smartphone. The approach based on hyperspectral imaging of pigmented tissue is also not suitable for implementation in a smart phone. Using A more 
promising approach would be to make use of the spectral information that is outside the visible spectral range (Neittaanmäki-Perttu et al. 2013), which would require thermal detector array rather than a CMOS imager in silicon. A more pragmatic approach would be analyse the visible/ near-IR spectrum in more detail, which would require a microspectrometer.

A second example that tries to provide an answer to the rising prevalence of skin cancer is the UV exposure-app. The objective is to monitor sunlight exposure and to provide a warning in case of too much UV exposure. Problem is that shorter-wavelength UV radiation is more harmful as compared to the longer wavelength components (http:// www.esrl.noaa.gov/gmd/grad/neubrew/docs/UVindex.pdf). Consequently, spectral weighing function is introduced in the definition of the UV index. The mere fact that UV light intensity is to be measured implies that the relevant part of the spectrum is well outside the spectral operating range of the in-phone imager. Therefore, most UV exposure apps are not actually measuring local UV exposure, but rather collect meteo information and provide these to the user. The Samsung Galaxy Note 4 does actually contain a UV sensor and the associated app (the $S$ health-app) provides 5 risk levels. However, the definition of the UV-index requires the measurement in at least three narrow spectral bands, which implies that the use of a single UV sensor could not be fully adequate. It should be noted that a simple set of three filters (rather than a full-fledged microspectrometer) could be sufficient.

A third example is about the measurement of the concentration of fine particles in air. Basically the horizon is imaged in a series of images, while moving the smartphone manually over a certain angle in elevation (http:// ispec.nl/). The spectrum and polarization of scattered light are analyzed. Apps are developed for that purpose, but also specific hardware is required that is to be placed in front of the imager. It seems somewhat unlikely that the limited size of this particular market would justify an effort for integrating this front-end with the imager within the smart phone. However, this example shows that specially designed hardware for spectral analysis in combination with the smart phone is an approach that has already been considered. This could be an interesting initial marketing strategy before introducing MEMS microspectrometers in smart phones.

\section{Beyond apps}

There are several high-volume applications for the CMOScompatible MEMS microspectrometer that are not related to the smart phone. A very promising application would be in point-of-care diagnostic instrumentation. This medical

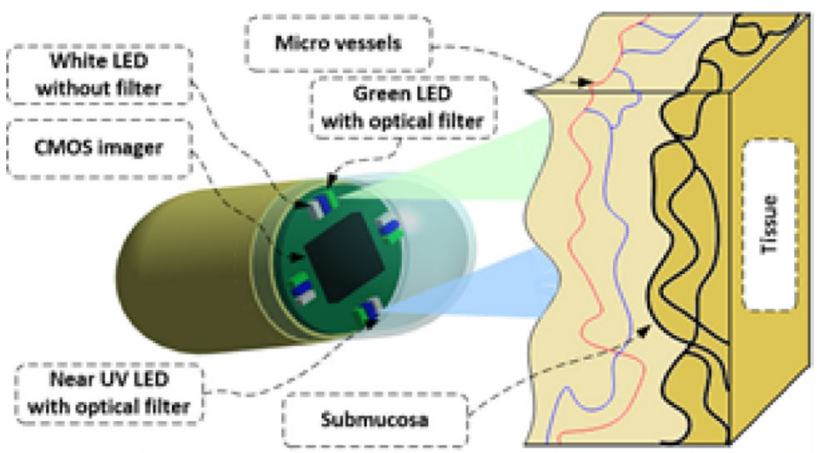

Fig. 3 Endoscopic capsule with narrowband imaging (Silva et al. 2014)

application area combines the high-volume advantage (many of such 'low-threshold nodes' are present in a typical infrastructure for patient care) with the need for small dimensions (which is essential, for instance, in implantable systems). The dimensions of devices such as the endoscopic capsule (also referred to as the 'smart pill'), which was introduced in 2000 for video endoscopy in the gastrointestinal tract, are by necessity highly limited (Qureshi 2004). Therefore, the dimensional advantage of the MEMS is in this case the key driver. The 'smart pill' is basically an imaging device traveling down the gastrointestinal tract, while a number of white LEDs are used for tissue illumination. An important improvement is the use of narrowband imaging. Well-defined green light and blue light is used for imaging of the more shallow and deeper parts of the tissue respectively, as is shown schematically in Fig. 3 (Silva et al. 2014). The narrowband imaging system is structurally compatible with existing imaging devices. White LEDs need to be exchanged for light sources that emit the prescribed spectra. This is merely filtering of the light source. Integration of a microspectrometer in the endoscopic capsule for photo luminescence studies of tissue would bring an important diagnostic capability to the system.

Many other promising medical applications are in the IR. One example is mentioned already is improving melanoma pre-screening by extending the spectral analysis into the IR (Neittaanmäki-Perttu et al. 2013). Opening this field of applications in the IR would require a technique for lowcost, CMOS-compatible fabrication of MEMS-based thermal detector arrays, which is one of the future trends in technology development.

There are several high-volume LVOF microspectrometer applications of high potential that are not directly related to the smart phone. A very promising application would be in gas analysis. The depletion of natural gas sources necessitates the introduction of sustainable energy source, such as biogas, to natural gas. Therefore, for safe and clean combustion, the composition of the gas fed into the burner 
needs to be known. This needs to be implemented at each user (i.e. burner, household), which implies a high-volume application. Therefore, gas sensors that are both reliable and low-cost gas are required. IR absorption spectroscopy using microspectrometers are suitable in this application (Ghaderi et al. 2014). The transmission spectra of the main components in natural gas are in the 3-3.7 $\mu \mathrm{m}$ wavelength range (Stein 2001). Therefore, the composition of the gas can in principle be measured by absorption spectroscopy in that part of the spectrum. Gas composition measurement is also being explored in the long-IR spectral range (Tuohiniemi et al. 2013). The gas chamber is typically the only part of the microspectrometer system used in absorption spectroscopy that is not miniaturized. The functionality of the detection chamber can be combined with that of an optical resonator operating at high order mode, when using the so-called lossy-FP microspectrometer. Such microsystems provide the ultimate integration density and are presently being investigated for future systems for gas absorption spectroscopy (Ayerden et al. 2014).

Another application field of great interest is in absorption spectroscopy of emission gases. Some have significant absorption peaks in the UV and short-wavelength to visible parts of the spectrum. However, the absorption cross section is typically much smaller as compared to absorption peaks in the IR. Therefore, a reduced signal level is to be expected at constant gas chamber volume. Moreover, suitable materials (i.e. sufficient index of refraction contrast and low absorption) for the design of optical filters and mirrors are less abundant in the UV spectral range, as compared to the visible. Air is investigated for that purpose as an 'optical material' with layer thickness defined by the airgap (Tuohiniemi et al. 2012; Ghaderi and Wolffenbuttel 2014).

\section{Conclusions}

The technology for CMOS-compatible MEMS microspectrometer fabrication has reached a sufficiently high level of maturity that fully-integrated microsystems can be considered. Trends are likely to be significantly driven by applications. Apart from highly specific applications, such as military, security and space, the markets are mainly to be found where the advantages of MEMS technology really makes a difference. These are characterized by either high-volume or extremely small dimensions. The LVOF-concept offers CMOS compatibility and allows the optical design of the microspectrometer to the specifications of the application by the design of the reflow mask.

Promising high-volume markets are those where instruments will be required in every household, because of some foreseeable trend (an example is absorption spectroscopy of combustible gas, because of the trend towards more flexible gas composition, or in exhaust gas analysis, because of more stringent emissions standards). The main field that is expected to drive microspectrometer developments is medicine. Personalized health using smart phone, which is already packed with sensors, is one of these, where applications are 'unlocked' using apps.

The medical application is also the main provider of applications in which small microsystem dimensions are paramount. This is especially the case in implantable devices and the smart pill. Point of care diagnostics is a medical application area in which both the low cost in high volume and the small system dimensions are decisive.

Acknowledgments This work has been supported by the Dutch technology foundation STW under Grant DEL.11476.

Open Access This article is distributed under the terms of the Creative Commons Attribution 4.0 International License (http://creativecommons.org/licenses/by/4.0/), which permits unrestricted use, distribution, and reproduction in any medium, provided you give appropriate credit to the original author(s) and the source, provide a link to the Creative Commons license, and indicate if changes were made.

\section{References}

Ayerden NP, Ghaderi M, de Graaf G, Wolffenbuttel RF (2014) A lossy Fabry-Perot based optical filter for natural gas analysis. Proc Eng 87:1410-1413

Correia JH, Bartek M, Wolffenbuttel RF (1999) Bulk micromachined tunable Fabry-Perot micro-interferometer for the visible spectral range. Sens Actuators A76:191-196

Das A, Swedish T, Wahi A, Moufarrej M, Noland M, Gurry T, ArandaMichel E, Aksel D, Wagh S, Sadashivaiah V, Zhang X, Raskar R (2015) Mobile phone based mini-spectrometer for rapid screening of skin cancer. SPIE Next generation spectroscopic technologies VIII. In: Druy M, Crocombe R (eds) Proc. SPIE, vol 9482, 94820M

Emadi A, Wu H, Grabarnik S, De Graaf G, Wolffenbuttel R (2009) Vertically tapered layers for optical applications fabricated using resist reflow. J Micromech Microeng 19:074014

Emadi A, Wu H, de Graaf G, Wolffenbuttel R (2012) Design and implementation of a sub-nm resolution microspectrometer based on a linear-variable optical filter. Opt Express 20:489-507

Ghaderi M, Wolffenbuttel RF (2014) Design and fabrication of multiple airgap-based visible filters. Proc SPIE 9130:913005

Ghaderi M, Ayerden NP, Emadi A, Enoksson P, Correia JH, de Graaf G, Wolffenbuttel RF (2014) Design, fabrication and characterization of infrared LVOFs for measuring gas composition. J Micromech Microeng 24:084001

Grabarnik S, Wolffenbuttel RF, Emadi A, Loktev M, Sokolova E, Vdovin G (2007) Planar double-grating microspectrometer. Opt Express 15(6):3581-3588

Grabarnik S, Emadi A, Wu H, de Graaf G, Wolffenbuttel RF (2008) High-resolution microspectrometer with an aberration-correcting planar grating. J Appl Opt 47(34):6442-6447

http://ec.europa.eu/research/participants/data/ref/h2020/ wp/2014_2015/main/h2020-wp1415-health_en.pdf

http://www.esrl.noaa.gov/gmd/grad/neubrew/docs/UVindex.pdf http://ispec.nl/ 
http://www.ncbi.nlm.nih.gov/pmc/articles/PMC3959919/

http://www.npr.org/sections/health-shots/2013/01/16/169524178/ skin-doctors-question-accuracy-of-apps-for-cancer-risk

http://www.peekvision.org/what-it-does (retina app)

http://www.regulations.gov/\#!documentDetail;D=FDA2011-D-0530-0001 (also http://www.fda.gov/downloads/ForConsumers/ConsumerUpdates/UCM263405.pdf)

http://www.sciencedaily.com/releases/2014/10/141020084751.htm

https://www.skinvision.com/en

Jerman JH, Clift DJ, Mallinson SR (1991) A miniature Fabry-Perot interferometer with a corrugated silicon diaphram support. Sens Actuators A29:151-158

Keller LD, Jaffe DT, Ershov OA, Benedict T, Graf UU (2000) Fabrication and testing of chemically micromachined silicon echelle gratings. Appl Opt 39(7):1094-1105

Lin C-H, Fong C-H, Lin Y-M, Lee Y-Y, Fung H-S, Shew B-Y (2011) EUV interferometric lithography and structural characterization of an EUV diffraction grating with non-destructive spectroscopic ellipsometry. Microelectronic Eng 88:2639-2643

Martin J, Krueger J, Gareau D (2014) Hyperspectral imaging for melanoma screening. Photonic therapeutics and diagnostics X. In: Choi B (ed) Proc. SPIE, vol 8926, p 892611

Mohr J, Anderer B, Ehrfeld W (1991) Fabrication of a planar grating spectrograph by deep-etch lithography with synchrotron radiation. Sens Actuators A25-27:571-575

Neittaanmäki-Perttu N, Gronroos M, Tani T, Polonen I, Ranki A, Saksela O, Snellman E (2013) Detecting field cancerization using a hyperspectral imaging system. Lasers Surg Med 45:410-417
Stein SE (2001) Infrared Spectra. In: Linstrom PJ and Mallard WG (eds) NIST chemistry WebBook, NIST standard reference database number 69. National Institute of Standards and Technology, Gaithersburg

Qureshi WA (2004) Current and future applications of the capsule camera. Nat Rev 3:447-450

Silva MF, Rodrigues JA, Oliveira MJ, Fernandes AR, Pereira S, Costa CG, Ghaderi M, Ayerden P, Goncalves LM, de Graaf G, Wolffenbuttel RF, Correia JH (2014) Optical filter for providing the required illumination to enable narrow band imaging. Proc Eng 87:1414-1417

Tanabe T, Notomi M, Kuramochi E, Shinya A, Taniyama H (2007) Trapping and delaying photons for one nanosecond in an ultrasmall high-Q photonic-crystal nanocavity. Nat Photonics 1(1):49-52

Tuohiniemi M, Blomberg M, Akujärvi A, Antila J, Saari H (2012) Optical transmission performance of a surface-micromachined Fabry-Pérot interferometer for thermal infrared. J Micromech Microeng 22:115004

Tuohiniemi M, Näsilä A, Mäkynen J (2013) Characterization of the tuning performance of a micromachined Fabry-Perot interferometer for thermal IR. J Micromech Microeng 23:075011

Wolffenbuttel RF (ed) (1996) Silicon sensors and circuits: on-chip compatibility. Chapman \& Hall, London

Wolffenbuttel RF (2005) MEMS-based optical mini- and microspectrometers for the visible and infrared spectral range. J Micromech Microeng 15:S145-S152 